\title{
Nephrotic syndrome in a patient with visceral leishmaniasis
}

\author{
T A D Tilakaratne ${ }^{1}$, I W G A L Malhasi ${ }^{1}$, A Rathnathilaka ${ }^{1}$
}

Ceylon Medical Journal 2018; 63: 31-32

DOI: http://doi.org/10.4038/cmj.v63i1.8622

\section{Introduction}

Visceral leishmaniasisis caused by the intramacropahage protozoa Leishmania. Since the first locally acquired case in 2007 a limited number of cases of visceral leishmaniasis have been diagnosed in comparison to the large number of cutaneous leishmaniasis cases in Sri Lanka [1]. Following an incubation period of 2-6 months patients have an insidious onset of clinical features with fever, weight loss, anaemia, organomegaly (hepatosplenomegaly) and oedema with renal involvement. It is newly identified as an AIDS related opportunistic infection. Common differential diagnosis of fever with nephrotic syndrome include systemic lupus erythematosis, lymphoma, leukaemia, renal, colonic or lung carcinoma, tuberculosis, syphilis, HIV and hepatitis B and C.

We report a patient who had been previously well presenting with a three month history of fever with nephrotic syndrome who was subsequently diagnosed with visceral leishmaniasis.

\section{Case report}

A 48 year old married female from Kalutara, returned after a two year stay in Dubai as a domestic helper because she had developed fever of one month duration. She was investigated at a local hospital for two months and later admitted to National Hospital of Sri Lanka. On admission she had fever, bilateral ankle swelling, with anemia and $3 \mathrm{~cm}$ firm non tender splenomegaly. There was no icterus, hepatomegaly or lymphadenopathy. Physical examination of the cardiovascular system, respiratory system and central nervous system were normal. She had nephrotic syndrome (urine protein to creatinine ratio 5.89),

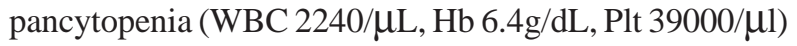
high ESR (84 mm) with ANA titer of 1:40. Renal biopsy was done at the local hospital considering a tentative diagnosis of SLE but it was not conclusive and repeat renal biopsy was not done due to severe pancytopenia. Meantime the patient developed rapid painful enlargement of spleen beyond the umbilicus three weeks after the initial admission. Splenic biopsy was performed. Light microscopy of splenic biopsy demonstrated leishmania amastigotes (Figure 1) which was confirmed with splenic biopsy culture and bone marrow culture. There was no evidence of amyloidosis in the spleen.

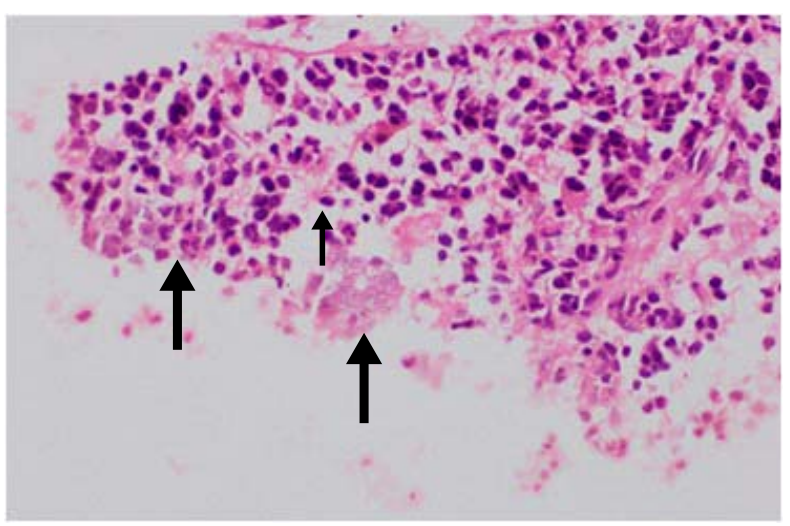

Figure 1. Splenic biopsy specimen in H \& E stain - magnification $400 x$

Thick arrows - multiple Leishmania donovani bodies within macrophages, Thin arrows - Plasma cells in background.

The rK39 assay was negative while dsDNA and tests for HIV, hepatitis B and C, brucellosis, malaria and repeat ANA were also negative. She was treated with Liposomal amphotericin B 3mg/Kg/day for 8 days. It was later withheld following a severe reaction to amphotericin.

She made a partial recovery as indicated by remission of fever spikes, reduced size of the spleen and haematological improvement. Parenteral nutrition and intravenous albumin were administered to prevent further hypoalbuminaemia. Routine splenectomy was planned but she refused surgery. At 11 weeks of hospital stay she

${ }^{1}$ National Hospital of Sri Lanka.

Correspondence: TADT, e-mail: <dilanka676@gmail.com>. Received 19 December 2017 and revised version accepted 05 January 2018. 
developed severe pain in the splenic area and ultrasound scan detected intrasplenic bleeding indicating urgent splenectomy. Post-operative period was complicated by extravasation of fluid into the peritoneum due to hypoproteinaemia and she died on post-operative day four in the surgical intensive care unit.

\section{Discussion}

Visceral leishmaniasis is considered an emerging infection in Sri Lanka. It is caused by Leishmania donovani [1]. About $40-60 \%$ of patients with visceral leishmaniasis have some form of renal involvement [2]. Visceral leishmaniasis affects both glomerular and tubular functions secondary to immune mediated injury as well as due to direct toxic effects by the pathogen itself. Clinical features range from asymptomatic proteinuria, pyuria, microscopic haematuria, mild to moderate renal impairment and acute kidney injury [2]. Renal impairment could be due to multiple causes such as anaemia, hypoalbuminaemia, hypovolaemia and direct renal toxicity. Histology specimens show tubulointerstitial, proliferative glomerulonephritis and proximal tubulopathy changes. Proteinuria in the range of $1-2 \mathrm{~g} / 24 \mathrm{hrs}$ are reported in the literature, but excretion rates of more than $2 \mathrm{~g} / 24 \mathrm{hrs}$ were not found in isolated cases of visceral leishmaniasis [3,4].

Severe nephrotic range proteinuria in visceral leishmaniasis is reported only when associated with either HIV or amyloidosis [4]. Our patient was negative for HIV. Her disease course was not long enough to cause amyloidosis and splenic biopsy stain was negative for amyloidosis. But our patient had severe proteinuria from the initial period of illness and it continued throughout the illness despite being negative for HIV or other chronic diseases. This has not been reported in the literature previously.
With no past or present features of cutaneous leishmaniasis the presence of proteinuria and joint symptoms could be suggestive of SLE. However concurrent SLE was unlikely due to the initial low titres ANA, negative DsDNA and a negative repeat ANA. As there was histological as well as culture confirmation our patient was treated for visceral leishmaniasis with severe complications. A number of studies have reported that antibodies that are found in SLE (eg. ANA, DsDNA) are positive in patients with visceral leishmaniasis too [5].

\section{Conclusion}

It is important that clinicians are aware that visceral leishmaniasis can present as a febrile illness with nephrotic syndrome. In spite of negative rk39 assay the possibility of visceral leishmaniasis should be considered in a patient with fever and nephrotic syndrome. This will result in early diagnosis and treatment.

\section{References}

1. Siriwardana HVYD, Chandrawansa PH, Sirimanna G, Karunaweera ND. Leishmaniasis in Sri Lanka: a decade old story. Sri Lanka J of Infect D 2012, 2(2): 2-12.

2. Clementi, Anna et al. Renal Involvement in Leishmaniasis: A Review of the Literature. NDT Plus 2011, 4.3: 147-52.

3. Dutra, Margarida et al. Renal Involvement in Visceral Leishmaniasis. Am J Kidney Dis, 1985; 6.1: 22-7.

4. Geraldo Bezerra da Silva Junior, Elvino José Guardão Barros, and Elizabeth De Francesco Daher, 'Kidney Involvement in Leishmaniasis - a Review', Braz J Infect Dis 2014, 18.4, 434-40.

5. Santana IU, Dias B, Nunes EA, Rocha FA, Silva FS, Jr, Santiago MB. Visceral leishmaniasis mimicking systemic lupus erythematosus: case series and a systematic literature review. Semin Arthritis Rheum 2015; 44: 658-65. 